\title{
Nitrogen Fixation and Nutritional Yield of Cowpea-Amaranth Intercrop
}

\author{
Buhlebelive Mndzebele ${ }^{1,2, *}$,, Bhekumthetho Ncube ${ }^{1}$, Melvin Nyathi ${ }^{1}$, Sheku Alfred Kanu ${ }^{3,4}$, \\ Melake Fessehazion ${ }^{1}$, Tafadzwanashe Mabhaudhi ${ }^{2}{ }^{(D}$, Stephen Amoo ${ }^{1}(\mathbb{D}$ \\ and Albert Thembinkosi Modi ${ }^{2}$ (D) \\ 1 Agricultural Research Council-Vegetable and Ornamental Plants, Private Bag X293, \\ Pretoria 0001, South Africa; bbkncube@yahoo.com (B.N.); MNyathi@arc.agri.za (M.N.); \\ fmelake@gmail.com (M.F.); AmooS@arc.agric.za (S.A.) \\ 2 Centre for Transformative Agricultural and Food Systems, School of Agricultural, Earth and Environmental \\ Sciences, College of Agriculture, Engineering and Science, University of KwaZulu-Natal, Private Bag X01, \\ Scottsville 3209, Pietermaritzburg, South Africa; mabhaudhi@ukzn.ac.za (T.M.); modiat@ukzn.ac.za (A.T.M.) \\ 3 College of Agriculture and Environmental Sciences, University of South Africa, P O Box 392, \\ Pretoria 0003, South Africa; kanu.popfred@gmail.com \\ 4 Department of Crop Science, Njala University, Freetown, Sierra Leone \\ * Correspondence: mndzebelebm@gmail.com; Tel.: +27-(0)-12-808-8000
}

Received: 11 March 2020; Accepted: 10 April 2020; Published: 15 April 2020

check for updates

\begin{abstract}
Nutrient-poor soils coupled with micronutrient deficiency among many rural resource-poor communities remain a challenge in sub-Saharan Africa. Nutrient-poor soils can be managed through various soil amendment or fertilisation strategies. Micronutrients can be supplied through plants. The study was aimed at determining the symbiotic nitrogen fixation of cowpea as well as the contribution of inter-cropping under varying levels of nitrogen, phosphorus, and potassium (NPK) fertilisation. In addition, the amount of micronutrients supplied by cowpea and amaranth were determined. The experiment was laid out in a $2 \times 4$ factorial treatment structure in a completely randomised design, with inter-cropping (cowpea and amaranth) and fertiliser (control, 25\%, 50\%, and $100 \%$ of the recommended NPK levels) as treatment factors with four replications. Symbiotic $\mathrm{N}_{2}$ fixation of cowpea decreased from $341-448 \mathrm{kgN} \cdot \mathrm{ha}^{-1}$ to $77-91 \mathrm{kgN}^{-h^{-1}}$ for the first year and 557-227 kgN.ha ${ }^{-1}$ to $92-164 \mathrm{kgN} \mathrm{ha}^{-1}$ for the second year with fertilisation. The iron and zinc nutritional yield increased (61-210 g.ha ${ }^{-1}$ for first year and 304-867 g.ha-1, for second year), proportional to fertiliser application to both crops. The research shows the benefits of leguminous crops in soil nutrient fertility and inorganic fertilisation with inter-cropping in managing micronutrient deficiency to meet the nutritional needs of rural communities.
\end{abstract}

Keywords: cowpea; amaranth; inter-cropping; $\mathrm{N}_{2}$ fixation; hidden hunger; nutritional yield

\section{Introduction}

Nutrient-poor soils and micronutrient deficiency (also known as "hidden hunger") are two interrelated challenges, affecting rural resource-poor farming communities in sub-Saharan Africa [1,2]. Hidden hunger is defined as a scenario where intake and absorption of minerals (in particular, essential micronutrients such as zinc and iron) are below amounts needed to maintain good health and development [3]. Poor soil fertility can be addressed through exogenous sources of fertilisation such as the application of organic/inorganic fertilisers and sometimes the use of leguminous crops through their symbiotic $\mathrm{N}_{2}$ fixation, which provide bio-fertilisers in cropping systems [4]. Low soil fertility affects crop production in rural resource-poor farming communities, reducing nutritional food 
security, leading to malnutrition [2,5]. Lack of nitrogen $(\mathrm{N})$, phosphorus $(\mathrm{P})$ and potassium $(\mathrm{K})(\mathrm{NPK})$ in sub-Saharan African soils tends to reduce micronutrient accumulation in plant tissues [6]. There is, in fact, a synergistic relationship between macro-mineral elements such as NPK in soils and trace element accumulation in plant tissues [7], hence the relatedness of the two factors. For example, $\mathrm{N}$ and $\mathrm{P}$ play an important role in the development of roots, which leads to acquisition, and subsequent translocation of nutrients to edible plant parts constituting economic yield [8]. It is, therefore, important to address these factors in combination due to an escalating rate of population growth which is estimated at 3.09\% per annum in Sub-Saharan Africa [9] and hence the need for additional micronutrients in human diets. When soils are low in macro-mineral nutrient elements, it can translate to reduced trace element accumulation in plant tissues, thus escalating "hidden hunger".

These measures, such as medicine based supplements, food enrichments or nutrients powder inclusion to meals are, however, inadequate to meet the increasing population's nutritional food needs, largely because of unaffordability [10]. In the quest to address nutritional food insecurity, food-based approaches involving vegetable production in smallholder farming systems are now employed as alternative solutions as they have been proven sustainable [11]. In addition, crop production approaches have also been confirmed effective in addressing household nutritional security [12].

Crop production approaches involve the growth of nitrogen-fixing leguminous crops, such as cowpea (Vigna unguiculata L. Walp), which is able to fix up to $337 \mathrm{~kg} \mathrm{~N} . ~ h a^{-1}$ [13]. In addition, cowpea can supply essential micronutrients [14], resulting in improved nutritional yield. When growing cowpea under subsistence farming system, some rural communities add, for example, inorganic fertiliser in addition to inter-cropping in order to optimise limited agricultural productive land [15]. Inter-cropping by rural resource-poor farmers dates back to ancient civilisation and is today still being practiced [16]. Some common inter-cropping systems entail a combination of a legume and non-legume crop [17]; for example, cowpea and amaranth (Amaranthus cruentus L.), both of which are utilised as indigenous vegetables. These indigenous vegetable crops are nutrient-dense particularly in crucial micro-elements such as iron and zinc [18]. Through inter-cropping, the physical, chemical and biological properties of the soil are improved, leading to increased crop growth and yield [19]. In order to measure the yield benefit of inter-cropping, several ratios are commonly used such as land equivalent ratio (LER). [20]. In addition, it has been shown that through inter-cropping (in consideration of benefit from two companion crops), there is more bioavailability of micro-nutrients in terms of concentration, when compared to sole cropping [21]. Micro-nutrient concentration becomes valuable to rural resource-poor communities especially in relation to available land and hence, the concept of nutritional yield (NY). Nutritional yield is defined as a unit of measure, which is a function of edible biomass (for example, leaves) and mineral nutritional composition in plant tissues [22].

There is a paucity of information on the link between symbiotic nitrogen fixation and fertilisation of mostly neglected indigenous vegetables such as cowpea and amaranth grown under inter-cropping system. Furthermore, the information on the effect of different fertilisation levels on nutritional yield of these crops is also limited. This study, therefore, hypothesised that (i) nitrogen fixation in cowpea will be affected by different fertiliser levels, and (ii) cowpea-amaranth intercrop will contribute to an improved NY compared to sole cropping of each crop. The objectives of the study were to assess the effect of fertiliser application and inter-cropping on the nodulation, nitrogen accumulation and symbiotic nitrogen fixation of cowpea. In addition, the effect of fertiliser application and inter-cropping on iron and zinc concentration, as well as nutritional yield of cowpea and amaranth were also assessed.

\section{Materials and Methods}

\subsection{Site Description and Environmental Conditions}

The experiment was conducted at the Agricultural Research Council (ARC), Vegetables and Ornamental Plant campus in Roodeplaat, Pretoria, South Africa $\left(25^{\circ} 35^{\prime} \mathrm{S}, 28^{\circ} 21^{\prime} \mathrm{E}, 1165\right.$ masl) during 2014/15 and 2015/16 summer seasons, from November to January as described by Mndzebele et al. [23]. 
The description of the soils in which the experimental sites were carried out, as well as the rainfall and temperature conditions during the trials were as detailed by Mndzebele et al. [23].

\subsection{Experimental Treatments, Layout and Plot Management}

The experimental treatments, layout and the management of the experiment were as described by Mndzebele et al. [23]. These entailed the amount of fertiliser applied, seedling preparation, planting, inter- and intra-row spacing as well as irrigation amounts applied.

Fertiliser (NPK) was applied based on the recommended cowpea requirement taking into consideration the lower fertiliser requirements of the legume. The experiment was laid out in a $2 \times 4$ factorial treatment structure in a completely randomised design (CRD) with four replications.

Irrigation scheduling was based on crop water requirement $\left(\mathrm{ET}_{\mathrm{c}}\right)$ of each crop, either in a sole cropping or in inter-cropping. Crop coefficient $\left(\mathrm{K}_{\mathrm{c}}\right)$ values for amaranth and cowpea were obtained from Beletse et al. [24].

\subsection{Data Collection}

\subsubsection{Determination of Symbiotic $\mathrm{N}_{2}$ Fixation in Cowpea}

To determine symbiotic $\mathrm{N}_{2}$ fixation, nodulation (number and mass of nodules formed), relative ureide- $\mathrm{N}(\% \mathrm{RU}-\mathrm{N})$, percentage $\mathrm{N}$ derived from $\mathrm{N}_{2}$ fixation (\%Ndfa), $\mathrm{N}$-fixed and soil $\mathrm{N}$ uptake of cowpea were determined. The relative ureide- $\mathrm{N}(\% \mathrm{RU}-\mathrm{N})$, percentage $\mathrm{N}$ derived from $\mathrm{N}_{2}$ fixation (\%Ndfa), $\mathrm{N}$-fixed and soil $\mathrm{N}$ uptake were computed as a factor of above-ground biomass shown in Mndzebele et al. [23]. The choice of the method was informed by the simplicity in the analyses and less costly equipment. Sampling was done for the ureides in the xylem sap, in which ureide $\mathrm{N}$ and nitrate $\mathrm{N}$ concentrations were obtained to calculate \%RU-N, \%Ndfa, N-fixed and soil $\mathrm{N}$ uptake in cowpea. The $\%$ Ndfa was determined using the calibration equation based on root-bleeding sap by Herridge and Peoples [25]. To determine the relative ureide- $\mathrm{N}$ abundance, equation 1 was used as shown in Table 1. The amount of N-fixed was calculated as Shearer and Kohl [26], as shown in Table 1. The total soil $\mathrm{N}$ uptake was calculated as indicated in Table 1. 
Table 1. Equations utilised to compute selected factors.

\begin{tabular}{|c|c|c|}
\hline Equations & Description & Number \\
\hline Relative ureide abundance (\%) & [ureide-N/ureide- $\mathrm{N}+$ nitrate-N] $\times 100$ & 1 \\
\hline N-fixed & $\%$ Ndfa $\times$ legume biomass-N; where, legume biomass $\mathrm{N}$ was the $\mathrm{N}$ content of AGB. & 2 \\
\hline The total soil $\mathrm{N}$ uptake & It was calculated by computing the difference between plant total $\mathrm{N}$ and $\mathrm{N}$-fixed. & 3 \\
\hline $\mathrm{NY}_{\text {cowpea }(\mathrm{Fe} \text { and } \mathrm{Zn})}=[\mathrm{MC} \times \mathrm{AGEB} \times 10]$ & 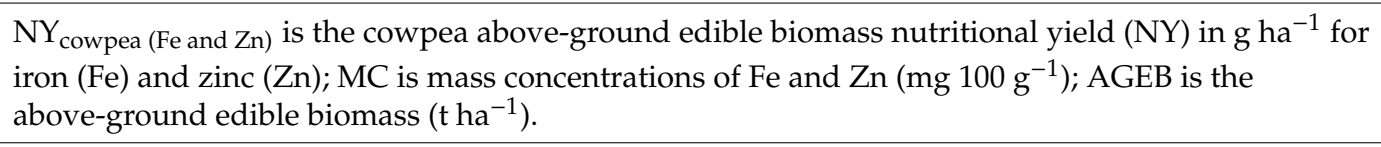 & 4 \\
\hline$N Y_{\text {amaranth }(\mathrm{Fe} \text { and } \mathrm{Zn})}=[\mathrm{MC} \times \mathrm{AGEB} \times 10]$ & $\begin{array}{l}\mathrm{NY} \text { amaranth (Fe and } \mathrm{Zn}) \text { is the amaranth above-ground edible biomass nutritional yield (NY) } \mathrm{g} \mathrm{ha}^{-1} \text { for } \\
\text { iron (Fe) and zinc }(\mathrm{Zn}) \text {; MC is mass concentrations of Fe and } \mathrm{Zn}\left(\mathrm{mg} 100 \mathrm{~g}^{-1}\right) \text {; AGEB is the } \\
\text { above-ground edible biomass }\left(\mathrm{t} \mathrm{ha}^{-1}\right) \text {. }\end{array}$ & 5 \\
\hline 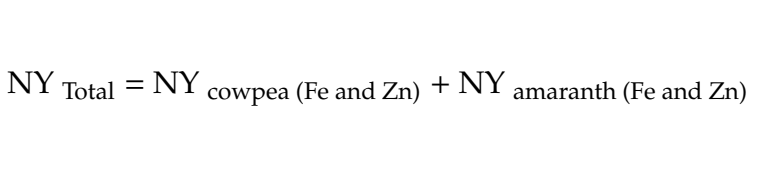 & 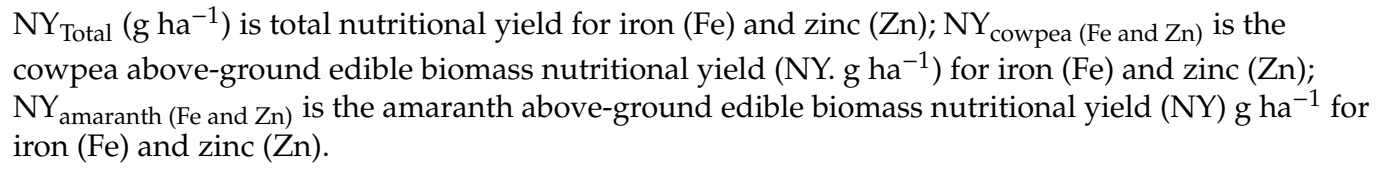 & 6 \\
\hline
\end{tabular}




\subsubsection{N-Accumulation, Ureide-and Tissue Nitrate-N of Cowpea at Physiological Maturity}

Shoots washed with distilled water to remove debris, were dried and analysed for mineral element concentration. The dried samples were digested using perchloric + nitric acid methods. A mass of $0.5 \mathrm{~g}$ of dry sample was digested in a $100 \mathrm{~mL}$ volumetric flask containing $7 \mathrm{~mL} \mathrm{HNO}_{3}$ (conc. nitric acid) and $3 \mathrm{~mL} \mathrm{HClO}_{4}$ (perchloric acid) at $180{ }^{\circ} \mathrm{C}$ [27]. The digested samples were analysed using the combustion method to determine the concentrations of the nitrogen element. The $\mathrm{N}$ content, indicating $\mathrm{N}$-accumulation of cowpea was calculated as a product of $\mathrm{N} \%$ and sample mass [28]. The sample mass to calculate $\mathrm{N}$-content was obtained from Mndzebele et al. [23]. The ureide- $\mathrm{N}$ and nitrate-N were analysed using the Rimini-Schryver reaction as described by Young and Conway [29], in Unkovich et al. [30]. Nitrates in cowpea were quantified using the salicylic acid method of Cataldo et al. [31], as outlined by Unkovich et al. [30].

\subsubsection{Measurement of Mineral Element Concentrations in Plant Tissue}

Dried leaf samples were analysed for mineral element concentration at the Agricultural Research Council-Soil, Climate, and Water (ARC-SCW) laboratory, Pretoria, South Africa. The dried samples were digested using perchloric + nitric acid methodology. A mass of $0.5 \mathrm{~g}$ of dry sample was digested in a $100 \mathrm{~mL}$ volumetric flask with $7 \mathrm{~mL} \mathrm{HNO}_{3}$ (concentrated nitric acid) and $3 \mathrm{~mL} \mathrm{HClO}_{4}$ (perchloric acid) at $180^{\circ} \mathrm{C}$ [27]. The digested samples were analysed using an Inductively Coupled Plasma-Optical Emission Spectrometry (ICP-OES) to determine the concentrations of the micronutrient elements (specifically Fe and Zn).

\subsubsection{Nutritional Yield}

Nutritional yield (NY) was calculated for cowpea and amaranth using equations 4 to 6 (Table 1). The computations included the concentrations of $\mathrm{Fe}$ and $\mathrm{Zn}$ and the above ground edible biomass (AGEB), obtained from Mndzebele et al. [23], for each crop. The total NY factoring both amaranth and cowpea were also determined as shown in equations 4 to 6 in Table 1.

\subsubsection{Statistical Analysis}

Data were subjected to a two-way analysis of variance with fertiliser levels and cropping system as the main factors. Data analysis was performed using GenStat statistical software (Version 19, VSN International, Hemel Hempstead, UK). Statistically significant treatment means were separated using Fisher least significant difference test at $p \leq 0.05$.

\section{Results}

\subsection{N-Accumulation, Ureide, and Tissue Nitrate-Nitrogen of Cowpea at Physiological Maturity}

There was a significant interaction between cropping systems and fertiliser levels with regard to $\mathrm{N} \%$, shoot $\mathrm{N}$-content, ureide and tissue $\mathrm{NO}_{3}$ concentration in cowpea tissues (Table 2). The highest $\mathrm{N} \%$ and shoot N-content were recorded at $25 \%$ NPK fertilisation in both-cropping systems, with the lowest obtained at $100 \%$ NPK (Table 2). Nitrogen accumulation (N\% and N-content) increased up to $50 \%$ NPK fertiliser level in the first season, in both cropping systems. The lowest $\mathrm{N}$-accumulation was observed in the control as well as 100\% NPK fertilisation treatments (Table 2). The highest ureide concentration was shown in the interaction of the control fertiliser level with inter-cropping. Ureide concentration showed an inverse proportion in response to fertilisation from control $(0 \%)$ treatment, up to $100 \%$ NPK fertiliser level (Table 2) in both seasons. There were significant $(p<0.05)$ interactions between cropping system and fertiliser level on tissue nitrate concentration in both seasons, with a characteristic increase as the fertiliser level increased up to $50 \%$ NPK and a sharp decline at $100 \%$ NPK although the concentrations were not significantly different from those of the control (Table 2). 
Table 2. Percentage N, shoot N-content, ureides and tissue $\mathrm{NO}_{3}$-concentration of cowpea fertilised with four different fertiliser (NPK) levels in 2014/15 (S1) and 2015/16 (S2) seasons.

\begin{tabular}{|c|c|c|c|c|c|}
\hline Cropping System [2014/15(S1)] & Fertiliser Level & $\mathbf{N}$ & Shoot N-Content & Ureide & $\begin{array}{l}\text { Tissue } \\
\mathrm{NO}_{3} \text {-Concentration }\end{array}$ \\
\hline & & $\%$ & g.plant ${ }^{-1}$ & $\mathrm{mM}$ & $\%$ \\
\hline \multirow{5}{*}{ Sole } & Fertiliser Levels & & & & \\
\hline & CONTROL & $5.3 \pm 0.1 \mathrm{a}^{1}$ & $2.6 \pm 0.18 b^{2}$ & $0.05 \pm 0.025 b^{1}$ & $1.5 \pm 0.17 \mathrm{~cd}^{3}$ \\
\hline & $25 \% \mathrm{NPK}$ & $5.4 \pm 0.2 \mathrm{a}^{1}$ & $4.0 \pm 0.30 \mathrm{a}^{1}$ & $0.02 \pm 0.002 \mathrm{~d}^{2}$ & $2.9 \pm 0.09 b^{2}$ \\
\hline & $50 \% \mathrm{NPK}$ & $4.7 \pm 0.2 \mathrm{a}^{1}$ & $4.3 \pm 0.28 \mathrm{a}^{1}$ & $0.02 \pm 0.003 \mathrm{~d}^{2}$ & $3.9 \pm 0.34 \mathrm{a}^{1}$ \\
\hline & $100 \%$ NPK & $4.7 \pm 0.3 \mathrm{a}^{1}$ & $3.6 \pm 0.29 a^{1}$ & $0.01 \pm 0.003 \mathrm{~d}^{2}$ & $1.2 \pm 0.04 \mathrm{~d}^{3}$ \\
\hline \multirow{4}{*}{ Intercrop } & CONTROL & $3.4 \pm 0.3 b^{2}$ & $1.6 \pm 0.24 b c^{2}$ & $0.06 \pm 0.013 \mathrm{a}^{1}$ & $2.7 \pm 0.25 b^{1}$ \\
\hline & $25 \%$ NPK & $4.9 \pm 0.3 \mathrm{a}^{1}$ & $3.5 \pm 0.11 \mathrm{a}^{1}$ & $0.05 \pm 0.011 b^{2}$ & $1.6 \pm 0.05 \mathrm{~cd}^{3}$ \\
\hline & $50 \% \mathrm{NPK}$ & $4.8 \pm 0.2 \mathrm{a}^{1}$ & $1.8 \pm 0.24 \mathrm{bc}^{2}$ & $0.03 \pm 0.005 c^{3}$ & $2.5 \pm 0.18 b^{12}$ \\
\hline & $100 \%$ NPK & $3.9 \pm 0.2 b^{2}$ & $1.0 \pm 0.02 c^{3}$ & $0.03 \pm 0.013 c^{3}$ & $1.8 \pm 0.12 \mathrm{c}^{2}$ \\
\hline LSD ( $p$-value) & Cropping System & $0.3(<0.001)$ & $0.39(<0.001)$ & $0.002(<0.001)$ & $0.28(0.155)$ \\
\hline LSD ( $p$-value) & Fertiliser Levels & $0.5(0.007)$ & $0.55(<0.001)$ & $0.003(<0.001)$ & $0.40(<0.001)$ \\
\hline LSD ( $p$-value) & Cropping System X Fertiliser Levels & $0.7(0.005)$ & $0.77(0.001)$ & $0.005(<0.001)$ & $0.56(<0.001)$ \\
\hline Cropping System [2015/16(S2)] & Fertiliser Levels & & & & \\
\hline \multirow{4}{*}{ 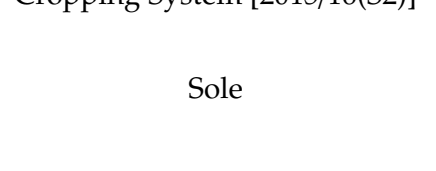 } & CONTROL & $3.5 \pm 0.1 b^{2}$ & $2.5 \pm 0.02 b^{2}$ & $0.052 \pm 0.024 b^{1}$ & $1.8 \pm 0.12 \mathrm{c}^{3}$ \\
\hline & $25 \% \mathrm{NPK}$ & $5.6 \pm 0.4 \mathrm{a}^{1}$ & $4.4 \pm 0.30 \mathrm{a}^{1}$ & $0.058 \pm 0.058 \mathrm{~b}^{1}$ & $3.5 \pm 0.54 b c^{2}$ \\
\hline & $50 \%$ NPK & $3.6 \pm 0.1 b^{2}$ & $2.9 \pm 0.10 b^{2}$ & $0.045 \pm 0.012 \mathrm{bc} c^{1}$ & $5.1 \pm 0.36 b^{1}$ \\
\hline & $100 \%$ NPK & $3.5 \pm 0.1 b^{2}$ & $1.8 \pm 0.08 \mathrm{~cd}^{3}$ & $0.046 \pm 0.004 b c^{1}$ & $1.3 \pm 0.14 c^{3}$ \\
\hline \multirow{4}{*}{ Intercrop } & CONTROL & $2.1 \pm 0.3 c^{2}$ & $1.4 \pm 0.20 \mathrm{~d}^{3}$ & $0.084 \pm 0.052 \mathrm{a}^{3}$ & $3.9 \pm 0.31 b c^{2}$ \\
\hline & $25 \%$ NPK & $3.5 \pm 0.2 b^{1}$ & $2.3 \pm 0.12 b c^{12}$ & $0.054 \pm 0.009 \mathrm{~b}^{3}$ & $5.7 \pm 1.38 b^{12}$ \\
\hline & $50 \% \mathrm{NPK}$ & $3.6 \pm 0.3 b^{1}$ & $2.8 \pm 0.38 b^{1}$ & $0.034 \pm 0.036 \mathrm{~cd}^{2}$ & $8.7 \pm 0.23 a^{1}$ \\
\hline & $100 \% \mathrm{NPK}$ & $3.9 \pm 0.2 b^{1}$ & $2.0 \pm 0.09 \mathrm{~cd}^{23}$ & $0.025 \pm 0.029 \mathrm{~d}^{1}$ & $1.9 \pm 0.15 c^{2}$ \\
\hline LSD ( $p$-value) & Cropping System & $0.4(<0.001)$ & $0.29(<0.001)$ & $0.007(0.774)$ & $1.24(0.002)$ \\
\hline LSD ( $p$-value) & Fertiliser Levels & $0.6(<0.001)$ & $0.40(<0.001)$ & $0.010(<0.001)$ & $1.76(<0.001)$ \\
\hline LSD ( $p$-value) & Cropping System X Fertiliser Levels & $0.8(0.002)$ & $0.57(<0.001)$ & $0.014(<0.001)$ & $2.48(0.387)$ \\
\hline
\end{tabular}

Mean \pm SE $(n=12)$ values in each column followed by different letters indicate significant differences between treatments (cropping system and fertiliser levels). Numerical values that have been superscripted compare means of each cropping system at different fertiliser levels $(p \leq 0.05)$. 


\subsection{Symbiotic $\mathrm{N}_{2}$ Fixation in Cowpea}

There was a significant $(p \leq 0.05)$ increase in nodulation (nodule biomass and number of nodules) of cowpea at physiological maturity in sole and inter-cropping when fertilisation was increased from control ( $0 \%$ NPK) up to $50 \%$ NPK, with an abrupt decline at $100 \%$ NPK (Table 3). The lowest nodulation was observed at the $100 \%$ NPK fertiliser level in both seasons, in sole and inter-cropping although not significantly different from those obtained at 25\% NPK (Table 3). Inter-cropping in combination with 50\% NPK fertilisation gave the highest nodule mass, in both seasons. In addition, the highest number of nodules was shown in sole-cropping in combination with $25 \%$ NPK fertilisation in both seasons. Cropping system comparison showed more nodule mass and the number of nodules in sole cropping relative to intercropping. The relative ureide- $\mathrm{N}(\% \mathrm{RU}-\mathrm{N})$ and the percentage $\mathrm{N}$ derived from $\mathrm{N}_{2}$ fixation (\%Ndfa) in cowpea was inversely proportional to the amount of fertiliser applied, with a significant decrease from control ( $0 \%$ ) to $100 \%$ NPK in both cropping systems (sole and inter-cropping) in both seasons (Table 3).

$\mathrm{N}$-fixed significantly decreased with the added amount of fertiliser application from the control ( $0 \%$ NPK) up to the $100 \%$ NPK in sole cropping system in the first season. However, there was an exception with the other treatment where there was a gradual increase from the control ( $0 \%$ NPK) until $25 \%$ NPK, which was the highest, while $100 \%$ NPK gave the lowest. There was a general characteristic increase in soil N-uptake with increase in fertilisation up to 50\% NPK in both cropping systems and in both seasons. On the other hand, inter-cropping recorded the highest soil $\mathrm{N}$-uptake in combination with $50 \%$ NPK in the second season (Table 3).

\subsection{Nutrient Concentration and Nutritional Yield in Cowpea and Amaranth}

Overall, there was a significant characteristic increase in iron $(\mathrm{Fe})$ and zinc $(\mathrm{Zn})$ concentration of cowpea and amaranth leaves as the fertiliser level increased from the control (0\%) to $100 \%$ NPK treatments in sole and inter-cropping and in both seasons (Table 4). Similarly, nutritional yield (Fe-NY, $\mathrm{Zn}-\mathrm{NY}$ and $\mathrm{Fe}+\mathrm{Zn}-\mathrm{NY}$ ) (Table 5) followed the same trend to that of the nutritional concentration except for the intercropping treatments, which showed an increase from $0 \%$ NPK to $25 \%$ NPK followed by a decline beyond these fertiliser levels for both crops in both seasons. 
Table 3. Nodule mass, number of nodules, \%RU-N, \%Ndfa, N-fixed and soil N uptake of cowpea fertilised with four different fertiliser (NPK) levels in 2014/15 and 2015/16 seasons.

\begin{tabular}{|c|c|c|c|c|c|c|c|}
\hline \multirow{2}{*}{ Cropping System [2014/15(S1)] } & \multirow[b]{2}{*}{ Fertiliser Levels } & \multirow{2}{*}{$\begin{array}{l}\text { Nodule Mass } \\
\text { g.plant }{ }^{-1}\end{array}$} & \multirow{2}{*}{$\begin{array}{l}\text { Nodule Number } \\
\text { per.plant }^{-1}\end{array}$} & \multirow{2}{*}{$\begin{array}{l}\text { RU-N } \\
\%\end{array}$} & \multirow{2}{*}{$\begin{array}{l}\% \text { Ndfa } \\
\%\end{array}$} & \multirow{2}{*}{$\begin{array}{l}\text { N-Fixed } \\
\text { kgN.ha }^{-1}\end{array}$} & \multirow{2}{*}{$\begin{array}{l}\text { Soil N-Uptake } \\
\text { kg.ha }^{-1}\end{array}$} \\
\hline & & & & & & & \\
\hline \multirow{4}{*}{ Sole } & CONTROL & $6.0 \pm 0.2 \mathrm{~d}^{3}$ & $54 \pm 4 b c^{12}$ & $75 \pm 2 a^{1}$ & $91 \pm 3 a^{1}$ & $341 \pm 23 b^{1}$ & $34 \pm 2 b^{2}$ \\
\hline & $25 \%$ NPK & $9.0 \pm 0.3 c^{2}$ & $99 \pm 5 a^{1}$ & $32 \pm 1 c^{2}$ & $24 \pm 2 c^{2}$ & $138 \pm 18 \mathrm{de}^{2}$ & $438 \pm 32 a^{1}$ \\
\hline & $50 \% \mathrm{NPK}$ & $11.3 \pm 0.6 a b^{1}$ & $59 \pm 4 b^{12}$ & $28 \pm 2 d^{3}$ & $17 \pm 1 \mathrm{~d}^{3}$ & $104 \pm 5$ def $^{23}$ & $509 \pm 37 a^{1}$ \\
\hline & $100 \% \mathrm{NPK}$ & $6.0 \pm 0.4 \mathrm{~d}^{3}$ & $27 \pm 1 c^{2}$ & $26 \pm 3 d^{3}$ & $15 \pm 1 d^{4}$ & $77 \pm 2 \mathrm{f}^{3}$ & $438 \pm 34 a^{1}$ \\
\hline \multirow{4}{*}{ Intercrop } & CONTROL & $6.0 \pm 0.4 \mathrm{~d}^{2}$ & $34 \pm 3 b c^{2}$ & $73 \pm 5 a^{1}$ & $88 \pm 4 a^{1}$ & $202 \pm 2 c^{2}$ & $28 \pm 3 b^{3}$ \\
\hline & $25 \%$ NPK & $10.3 \pm 0.5 b c^{1}$ & $30 \pm 2 b c^{2}$ & $73 \pm 3 a^{1}$ & $88 \pm 2 a^{1}$ & $448 \pm 17 a^{1}$ & $61 \pm 3 b^{2}$ \\
\hline & $50 \%$ NPK & $12.7 \pm 0.5 \mathrm{a}^{1}$ & $43 \pm 2 b c^{1}$ & $54 \pm 3 b^{2}$ & $57 \pm 5 b^{2}$ & $149 \pm 2 d^{3}$ & $113 \pm 11 b^{1}$ \\
\hline & $100 \% \mathrm{NPK}$ & $5.0 \pm 0.4 \mathrm{~d}^{2}$ & $35 \pm 2 b c^{12}$ & $53 \pm 5 b^{2}$ & $61 \pm 1 b^{2}$ & $91 \pm 9 \mathrm{ef}^{4}$ & $58 \pm 4 b^{2}$ \\
\hline LSD ( $p$-value) & C. System & $1.1(0.426)$ & $14(0.003)$ & $2(<0.001)$ & $3(<0.001)$ & $23(<0.001)$ & $45(<0.001)$ \\
\hline LSD ( $p$-value) & F. Levels & $1.5(<0.001)$ & $20(0.023)$ & $3(<0.001)$ & $4(<0.001)$ & $33(<0.001)$ & $64(<0.001)$ \\
\hline LSD ( $p$-value) & C. System X F. Levels & $2.2(0.330)$ & $28(0.008)$ & $4(<0.001)$ & $6(<0.001)$ & $47(<0.001)$ & $90(<0.001)$ \\
\hline \multirow{2}{*}{ Cropping System [2015/16(S2)] } & Fertiliser Levels & & & & & & \\
\hline & CONTROL & $3.7 \pm 0.3 \mathrm{de}^{3}$ & $42 \pm 4 b c^{12}$ & $74 \pm 1 \mathrm{a}^{1}$ & $88 \pm 2 a^{1}$ & $319 \pm 3 b c d^{2}$ & $43 \pm 2 \mathrm{~cd}^{2}$ \\
\hline \multirow{3}{*}{ Sole } & $25 \% \mathrm{NPK}$ & $6.0 \pm 0.4 b d^{2}$ & $76 \pm 4 a^{1}$ & $74 \pm 1 \mathrm{a}^{1}$ & $89 \pm 2 \mathrm{a}^{1}$ & $557 \pm 2 \mathrm{a}^{1}$ & $69 \pm 5 c d^{2}$ \\
\hline & $50 \% \mathrm{NPK}$ & $8.3 \pm 0.7 a b c^{1}$ & $45 \pm 2 b^{12}$ & $68 \pm 2 \mathrm{ab}^{1}$ & $80 \pm 2 \mathrm{ab}^{1}$ & $330 \pm 5 b^{2}$ & $82 \pm 3 b^{1}$ \\
\hline & $100 \% \mathrm{NPK}$ & $3.0 \pm 0.6 \mathrm{e}^{3}$ & $21 \pm 1 c^{2}$ & $56 \pm 3 c d^{2}$ & $62 \pm 2 \mathrm{~cd}^{2}$ & $164 \pm 4 b c^{2}$ & $101 \pm 2 b^{1}$ \\
\hline \multirow{4}{*}{ Intercrop } & CONTROL & $4.0 \pm 0.5 \mathrm{de}^{2}$ & $23 \pm 2 b c^{2}$ & $76 \pm 3 a^{1}$ & $92 \pm 4 \mathrm{a}^{1}$ & $185 \pm 17 \mathrm{~cd}^{1}$ & $16 \pm 2 d^{3}$ \\
\hline & $25 \%$ NPK & $8.3 \pm 0.6 b^{1}$ & $26 \pm 2 b c^{2}$ & $62 \pm 2 b c^{2}$ & $70 \pm 3 b c^{2}$ & $227 \pm 6 b c d^{1}$ & $97 \pm 6 b c^{23}$ \\
\hline & $50 \%$ NPK & $10.7 \pm 0.5 \mathrm{a}^{1}$ & $33 \pm 2 b c^{1}$ & $50 \pm 1 d^{3}$ & $51 \pm 2 d^{3}$ & $207 \pm 3 d^{1}$ & $199 \pm 3 b^{1}$ \\
\hline & $100 \% \mathrm{NPK}$ & $3.0 \pm 0.5 \mathrm{e}^{2}$ & $27 \pm 2 b c^{12}$ & $37 \pm 1 \mathrm{e}^{4}$ & $32 \pm 2 e^{4}$ & $92 \pm 6 d^{1}$ & $195 \pm 12 a^{2}$ \\
\hline LSD ( $p$-value) & C. System & $1.1(0.033)$ & $11(0.003)$ & $5(<0.001)$ & $8(<0.001)$ & $48(<0.001)$ & $34(0.015)$ \\
\hline LSD ( $p$-value) & F. Levels & $1.6(<0.001)$ & $16(0.023)$ & $7(<0.001)$ & $11(<0.001)$ & $69(<0.001)$ & $48(<0.001)$ \\
\hline LSD ( $p$-value) & C. System X F. Levels & $2.3(0.279)$ & $22(0.008)$ & $10(0.006)$ & $16(0.006)$ & $97(0.059)$ & $68(0.007)$ \\
\hline
\end{tabular}

Mean \pm SE $(n=12)$ values in each column followed by different letters indicate significant differences between treatments (cropping system and fertiliser levels). Numerical values that 
Table 4. Moisture content, iron mass concentration (Fe) and zinc mass concentration (Zn) of cowpea and amaranth under sole and inter-cropping systems at four fertiliser (NPK) levels in 2014/15 and 2015/16 summer seasons.

\begin{tabular}{|c|c|c|c|c|c|c|c|}
\hline \multirow[t]{3}{*}{ Cropping System [2014/15(S1)] } & & \multicolumn{3}{|l|}{ Cowpea } & \multicolumn{3}{|l|}{ Amaranth } \\
\hline & & Moisture Content & $\mathrm{Fe}$ & $\mathrm{Zn}$ & Moisture Content & $\mathrm{Fe}$ & Zn \\
\hline & Fertiliser Levels & & mg.100g-1 & mg.100g-1 & & mg.100g-1 & mg.100g-1 \\
\hline \multirow{4}{*}{ Sole } & CONTROL & $0.869 \pm 0.006 \mathrm{~d}^{3}$ & $25.7 \pm 1.5 d^{4}$ & $2.2 \pm 0.1 \mathrm{e}^{3}$ & $0.908 \pm 0.006 b^{1}$ & $41.9 \pm 1.7 c^{2}$ & $3.1 \pm 0.03 \mathrm{a}^{1}$ \\
\hline & $25 \% \mathrm{NPK}$ & $0.908 \pm 0.006 b^{2}$ & $60.4 \pm 1.0 c^{3}$ & $3.4 \pm 0.1 \mathrm{~cd}^{2}$ & $0.916 \pm 0.005 \mathrm{ab}^{1}$ & $44.3 \pm 4.2 \mathrm{bc}{ }^{12}$ & $3.1 \pm 0.16 \mathrm{a}^{1}$ \\
\hline & $50 \% \mathrm{NPK}$ & $0.919 \pm 0.006 a b^{12}$ & $75.1 \pm 5.3 b^{2}$ & $3.6 \pm 0.1 b c d^{2}$ & $0.918 \pm 0.005 \mathrm{ab}^{1}$ & $45.7 \pm 1.5 b c^{12}$ & $3.5 \pm 0.33 \mathrm{a}^{1}$ \\
\hline & $100 \% \mathrm{NPK}$ & $0.927 \pm 0.004 \mathrm{a}^{1}$ & $92.3 \pm 4.1 \mathrm{a}^{1}$ & $4.4 \pm 0.1 \mathrm{a}^{1}$ & $0.931 \pm 0.013 \mathrm{ab}{ }^{1}$ & $54.8 \pm 4.0 \mathrm{ab}^{1}$ & $3.7 \pm 0.34 \mathrm{a}^{1}$ \\
\hline \multirow{4}{*}{ Intercrop } & CONTROL & $0.843 \pm 0.008 \mathrm{e}^{3}$ & $23.7 \pm 1.4 \mathrm{~d}^{3}$ & $3.3 \pm 0.1 \mathrm{~d}^{2}$ & $0.912 \pm 0.008 \mathrm{ab}^{1}$ & $41.9 \pm 1.7 \mathrm{c}^{2}$ & $3.1 \pm 0.08 \mathrm{a}^{1}$ \\
\hline & $25 \%$ NPK & $0.847 \pm 0.008 \mathrm{e}^{3}$ & $24.1 \pm 1.4 \mathrm{~d}^{3}$ & $3.3 \pm 0.1 \mathrm{~d}^{2}$ & $0.917 \pm 0.010 \mathrm{ab}^{1}$ & $48.0 \pm 2.1 b c^{2}$ & $3.2 \pm 0.16 \mathrm{a}^{1}$ \\
\hline & $50 \% \mathrm{NPK}$ & $0.890 \pm 0.008 c^{2}$ & $52.3 \pm 1.6 c^{2}$ & $3.8 \pm 0.1 b c^{1}$ & $0.930 \pm 0.005 \mathrm{ab}^{1}$ & $54.8 \pm 4.0 \mathrm{ab}^{12}$ & $3.5 \pm 0.33 a^{1}$ \\
\hline & $100 \% \mathrm{NPK}$ & $0.917 \pm 0.005 \mathrm{ab}^{1}$ & $68.9 \pm 3.5 b^{1}$ & $4.0 \pm 0.1 \mathrm{ab}^{1}$ & $0.939 \pm 0.011 \mathrm{a}^{1}$ & $63.6 \pm 7.7 \mathrm{a}^{1}$ & $3.7 \pm 0.34 \mathrm{a}^{1}$ \\
\hline LSD ( $p$-value) & Cropping System & $0.02(<0.001)$ & $4.90(<0.001)$ & $0.270(0.056)$ & $0.02(0.319$ & $6.18(0.060)$ & $0.1(0.922)$ \\
\hline LSD ( $p$-value) & Fertiliser Levels & $0.01(<0.001)$ & $6.11(<0.001)$ & $0.282(<0.001)$ & $0.02(0.040$ & $8.14(0.001)$ & $0.5(0.022)$ \\
\hline LSD ( $p$-value) & $\begin{array}{l}\text { Cropping System } \\
\text { X Fertiliser Levels }\end{array}$ & $0.01(0.001)$ & $8.07(<0.001)$ & $0.385(<0.001)$ & $0.02(0.896$ & $10.96(0.594)$ & $0.6(1.000)$ \\
\hline \multirow[t]{2}{*}{ Cropping System [2015/16(S2)] } & Fertiliser Levels & & & & & & \\
\hline & CONTROL & $0.816 \pm 0.005 b^{1}$ & $66.5 \pm 3.0 c^{3}$ & $3.1 \pm 0.1 c^{2}$ & $0.903 \pm 0.009 a b^{1}$ & $64.0 \pm 2.6 \mathrm{e}^{4}$ & $\begin{array}{l}4.0 \pm 0 ., 4 \mathrm{bcd} \\
2\end{array}$ \\
\hline \multirow[t]{4}{*}{ Sole } & $25 \% \mathrm{NPK}$ & $0.806 \pm 0.006 \mathrm{~b}^{1}$ & $82.0 \pm 5.7 b^{23}$ & $3.1 \pm 0.2 c^{2}$ & $0.908 \pm 0.005 \mathrm{ab}{ }^{1}$ & $77.8 \pm 4.4 \mathrm{~d}^{3}$ & $4.1 \pm 0.2 b c d^{2}$ \\
\hline & $50 \% \mathrm{NPK}$ & $0.826 \pm 0.009 a b^{1}$ & $91.7 \pm 3.3 b^{2}$ & $3.4 \pm 0.1 b c^{12}$ & $0.911 \pm 0.006 \mathrm{ab}^{1}$ & $90.9 \pm 2.3 c^{2}$ & $4.8 \pm 0.2 \mathrm{ab}^{12}$ \\
\hline & $100 \%$ NPK & $0.811 \pm 0.007 \mathrm{~b}^{1}$ & $141.5 \pm 10.4 \mathrm{a}^{1}$ & $3.7 \pm 0.2 \mathrm{ab}^{1}$ & $0.913 \pm 0.006 \mathrm{ab}^{1}$ & $110.5 \pm 1.6 b^{1}$ & $5.6 \pm 0.5 \mathrm{a}^{1}$ \\
\hline & CONTROL & $0.804 \pm 0.003 b c^{2}$ & $67.5 \pm 1.8 c^{3}$ & $3.2 \pm 0.3 c^{2}$ & $0.896 \pm 0.008 b^{2}$ & $79.5 \pm 2.7 \mathrm{~d}^{3}$ & $3.1 \pm 0.2 \mathrm{~d}^{3}$ \\
\hline \multirow{3}{*}{ Intercrop } & $25 \% \mathrm{NPK}$ & $0.841 \pm 0.007 \mathrm{a}^{1}$ & $82.8 \pm 5.8 b^{2}$ & $3.2 \pm 0.1 b c^{2}$ & $0.911 \pm 0.003 a b^{12}$ & $85.1 \pm 2.3 \mathrm{~cd}^{3}$ & $3.5 \pm 0.3 c d^{23}$ \\
\hline & $50 \% \mathrm{NPK}$ & $0.784 \pm 0.009 c^{3}$ & $84.3 \pm 4.6 b^{2}$ & $3.5 \pm 0.2 \mathrm{abc} c^{12}$ & $0.913 \pm 0.009 a b^{12}$ & $106.2 \pm 2.6 b^{2}$ & $3.8 \pm 0.3 b c d^{2}$ \\
\hline & $100 \% \mathrm{NPK}$ & $0.752 \pm 0.016 \mathrm{~d}^{4}$ & $95.3 \pm 2.5 b^{1}$ & $3.9 \pm 0.1 \mathrm{a}^{1}$ & $0.917 \pm 0.005 \mathrm{a}^{1}$ & $129.5 \pm 3.7 \mathrm{a}^{1}$ & $4.4 \pm 0.3 b c^{1}$ \\
\hline LSD ( $p$-value) & Cropping System & $0.02(0.918)$ & $9.78(<0.001)$ & $0.20(0.001)$ & $0.02(0.918)$ & $9.33(<0.001)$ & $1.6(0.001)$ \\
\hline LSD ( $p$-value) & Fertiliser Levels & $0.01(0.100)$ & $10.11(<0.001)$ & $0.32(0.002)$ & $0.01(0.100)$ & $5.46(<0.001)$ & $0.4(0.002)$ \\
\hline LSD ( $p$-value) & $\begin{array}{l}\text { Cropping System } \\
\text { X Fertiliser Levels }\end{array}$ & $0.02(0.792)$ & $13.86(0.258)$ & $0.41(0.871)$ & $0.02(0.792)$ & $9.47(0.258)$ & $1.5(0.871)$ \\
\hline
\end{tabular}

Mean \pm SE $(n=12)$ values in each column followed by different letters indicate significant differences between treatments (cropping system and fertiliser levels). Numerical values that have been superscripted compare means of each cropping system at different fertiliser levels $(p \leq 0.05)$. 
Table 5. Iron nutritional yield (Fe-NY), Zinc nutritional yield (Zn-NY) and combined zinc and iron nutritional yield (Fe+Zn-NY) of cowpea and amaranth under sole and inter-cropping systems at four fertiliser (NPK) levels in 2014/15 and 2015/16 summer seasons.

\begin{tabular}{|c|c|c|c|c|}
\hline Crops (2014/15) & Fertiliser Level & Fe-NY & $\mathrm{Zn}-\mathrm{NY}$ & $\mathrm{Zn+Fe-NY}$ \\
\hline & & \multicolumn{3}{|c|}{ g.ha-1 } \\
\hline \multirow{4}{*}{ Amaranth } & CONTROL & $48 \pm 2 \operatorname{efg}^{2}$ & $3.5 \pm 0.5 \mathrm{~h}^{3}$ & $52 \pm 2 \mathrm{ef}^{2}$ \\
\hline & $25 \% \mathrm{NPK}$ & $76 \pm 4 \mathrm{ef}^{2}$ & $5.3 \pm 0.9 \mathrm{gh}^{23}$ & $81 \pm 4 \mathrm{ef}^{2}$ \\
\hline & $50 \%$ NPK & $97 \pm 3 e^{2}$ & $7.7 \pm 0.8 \mathrm{~g}^{2}$ & $105 \pm 3 e^{2}$ \\
\hline & $100 \% \mathrm{NPK}$ & $197 \pm 1 \mathrm{~cd}{ }^{1}$ & $13.3 \pm 1.3 \mathrm{ef}^{1}$ & $210 \pm 1 \mathrm{~d}^{1}$ \\
\hline \multirow{4}{*}{ Cowpea } & CONTROL & $19 \pm 1 g^{2}$ & $12.4 \pm 0.3 \mathrm{f}^{3}$ & $31 \pm 1 f^{2}$ \\
\hline & $25 \%$ NPK & $31 \pm 3 \mathrm{fg}^{1}$ & $21.4 \pm 1.6 c^{1}$ & $53 \pm 3 \mathrm{ef}^{1}$ \\
\hline & $50 \% \mathrm{NPK}$ & $33 \pm 1 \mathrm{fg}^{1}$ & $16.6 \pm 1.7 \mathrm{de}^{2}$ & $50 \pm 1 \mathrm{f}^{1}$ \\
\hline & $100 \%$ NPK & $41 \pm 2 \mathrm{fg}^{1}$ & $20.0 \pm 8 \mathrm{~cd}^{12}$ & $61 \pm 2 \mathrm{ef}^{1}$ \\
\hline \multirow[t]{4}{*}{ Amaranth and Cowpea } & CONTROL & $172 \pm 14 d^{3}$ & $21.0 \pm 1.8 \mathrm{c}^{3}$ & $193 \pm 13 d^{3}$ \\
\hline & $25 \%$ NPK & $234 \pm 34 c^{3}$ & $28.2 \pm 1.7 \mathrm{~b}^{2}$ & $262 \pm 35 c^{3}$ \\
\hline & $50 \% \mathrm{NPK}$ & $514 \pm 22 b^{2}$ & $36.5 \pm 2.4 \mathrm{a}^{1}$ & $550 \pm 21 b^{2}$ \\
\hline & $100 \%$ NPK & $605 \pm 20 \mathrm{a}^{1}$ & $35.5 \pm 2.1 \mathrm{a}^{1}$ & $641 \pm 21 \mathrm{a}^{1}$ \\
\hline LSD ( $p$-value) & Crops & $28(<0.001)$ & $1.9(<0.001)$ & $29(<0.001)$ \\
\hline LSD ( $p$-value) & Fertiliser Level & $32(<0.001)$ & $2.2(0.003)$ & $33(<0.001)$ \\
\hline LSD ( $p$-value) & Cropping System X Fertiliser Level & $55(<0.001)$ & $3.8(0.588)$ & $58(<0.001)$ \\
\hline \multicolumn{5}{|l|}{ Crops (2015/16) } \\
\hline \multirow{4}{*}{ Amaranth } & CONTROL & $67 \pm 6 h^{3}$ & $4.2 \pm \mathrm{g}^{3}$ & $71 \pm 6 h^{3}$ \\
\hline & $25 \% \mathrm{NPK}$ & $89 \pm 4 h^{3}$ & $4.7 \pm \mathrm{g}^{3}$ & $94 \pm 4 h^{3}$ \\
\hline & $50 \% \mathrm{NPK}$ & $162 \pm 7 \mathrm{gh}^{2}$ & $8.5 \pm \mathrm{fg}^{2}$ & $170 \pm 7 \mathrm{gh}^{2}$ \\
\hline & $100 \%$ NPK & $290 \pm 3 g^{1}$ & $14.5 \pm \mathrm{f}^{1}$ & $304 \pm 3 g^{1}$ \\
\hline \multirow[t]{4}{*}{ Cowpea } & CONTROL & $857 \pm 12 \mathrm{def}^{3}$ & $39.5 \pm 2.3 \mathrm{~cd}^{2}$ & $896 \pm 12 \mathrm{def}^{3}$ \\
\hline & $25 \% \mathrm{NPK}$ & $1617 \pm 9 a^{1}$ & $61.4 \pm 3.2 \mathrm{a}^{1}$ & $1678 \pm 9 a^{1}$ \\
\hline & $50 \% \mathrm{NPK}$ & $1298 \pm 16 b^{2}$ & $47.5 \pm 2.5 \mathrm{~b}^{2}$ & $1346 \pm 16 b^{2}$ \\
\hline & $100 \%$-NPK & $1084 \pm 13 c^{23}$ & $28.3 \pm 2.6 \mathrm{e}^{3}$ & $1112 \pm 13 c^{23}$ \\
\hline \multirow[t]{4}{*}{ Amaranth and Cowpea } & CONTROL & $770 \pm 16 f^{2}$ & $35.9 \pm 2.8 \mathrm{~cd}^{12}$ & $805 \pm 17 f^{2}$ \\
\hline & $25 \%$ NPK & $1025 \pm 28 c d^{1}$ & $40.7 \pm 2.2 c^{1}$ & $1066 \pm 28 \mathrm{~cd}{ }^{1}$ \\
\hline & $50 \%$ NPK & $962 \pm 27 c d^{1}$ & $39.5 \pm 2.9 \mathrm{~cd}^{1}$ & $1001 \pm 28 \mathrm{cde}^{1}$ \\
\hline & $100 \%$ NPK & $835 \pm 12 \mathrm{ef}^{2}$ & $32.8 \pm 2.5 \mathrm{de}^{12}$ & $867 \pm 12 \mathrm{ef}^{2}$ \\
\hline LSD ( $p$-value) & Crops & $78(<0.001)$ & $4.1(<0.001)$ & $78(<0.001)$ \\
\hline LSD ( $p$-value) & Fertiliser Levels & $90(<0.001)$ & $4.7(<0.001)$ & $90(<0.001)$ \\
\hline LSD ( $p$-value) & Crops $\times$ Fertiliser Levels & $155(<0.001)$ & $8.2(<0.001)$ & $156(<0.001)$ \\
\hline
\end{tabular}

Mean \pm SE $(n=18)$ values in each column followed by different letters indicate significant differences between treatments (crops and fertiliser levels). Numerical values that have been superscripted compare means of each cropping system at different fertiliser levels $(p \leq 0.05)$. 


\section{Discussion}

This study hypothesised that (i) nitrogen fixation in cowpea will be affected by different fertiliser levels, and (ii) cowpea-amaranth intercrop will contribute to improved NY compared to sole cropping of each crop. The objectives of the study were therefore to assess the effect of fertiliser application and inter-cropping on the nodulation, nitrogen accumulation as well as symbiotic nitrogen fixation of cowpea. In addition, iron and zinc concentration, nutritional yields of cowpea and amaranth were also assessed.

Nitrogen accumulation indicators include among others, the ureide-N and nitrate-N. The ureide- $\mathrm{N}$ was markedly higher in control treatments and decreased with increasing NPK fertiliser application treatments (Table 2). On the other hand, nitrate-N was significantly enhanced by an increased application of fertiliser treatment with the highest at 25\% NPK and 50\% NPK and lowest in cowpea grown with 100\% NPK fertiliser (Table 2). The overall results of this study indicated that both the ureide-and nitrate-N decreased by the application of $100 \%$ NPK. This demonstrates that the application of the highest NPK suppressed ureide- N and nitrate-N concentrations in cowpea, regardless of the cropping system, sole crop or intercropped with amaranth. Studies have shown that the application of higher levels of NPK in legumes tends to suppress the ureide- $\mathrm{N}$ and nitrate-N concentrations [32]. All these $\mathrm{N}$-accumulation processes lead to biomass accrual by cowpea, and plant growth. In general, the co-application of these nutrient elements at optimum concentrations increased the uptake and accumulation of N in crops [33]. Overall, the application of NPK at optimum concentrations results in synergistic interaction and promote not only the growth but the uptake and accumulation of $\mathrm{N}$ in plant tissue [7], as found in this study.

Symbiotic performance of cowpea indicated by nodulation as well as $\mathrm{N}_{2}$ fixation showed some degree of sensitivity to both fertilisation [34] and inter-cropping [35]. The higher nodulation (number of nodules and nodule mass) at physiological maturity in cowpea in treatments with fertiliser levels below the recommended rate (Table 3) could be attributed to the application of the $25 \%$ of NPK, which probably served as starter $\mathrm{N}, \mathrm{P}$, and $\mathrm{K}$, and was therefore crucial in stimulating nodule formation for symbiotic functioning [36]. By contrast, the study showed the least nodulation in cowpea, occurring at $100 \%$ NPK (Table 3), which somewhat confirms the assertion that higher mineral NPK levels tend to inhibit nodule formation in legumes [37]. Other studies have also indicated the sensitivity of nodulation to fertiliser application [37]. Generally, there is greater nodulation in the sole cropping [38] when compared to inter-cropping which shows lower amounts [39]. The reduced nodulation observed in the inter-cropping system (in consideration of individual crops), could have been caused by cowpea competition with amaranth, especially for below-ground resources. Inter-cropping results in competition between crops due to proximity with the companion crop [40], thus, resulting in low nodulation as obtained in this study. Higher nodulation in fertiliser treatments below the $100 \%$ NPK are perhaps an indication that the soil only required some level of NPK for the cowpea crop to kick start symbiotic performance (Table 3), in which root nodules function effectively. Indications through other studies have shown that increased fertiliser amounts inhibit nodulation [41]. This study satisfies the hypothesis that nitrogen fixation in cowpea will be higher at low fertiliser amounts as shown by the results.

The results also indicated the dependence on atmospheric $\mathrm{N}_{2}(\% \mathrm{Ndfa})$ and $\mathrm{N}$-fixed in cowpea at physiological maturity showed an inverse relation to additional fertilisation in both seasons (Table 3). According to Anglade et al. [42], legumes tend to increase their \%Ndfa, whilst decreasing $\mathrm{N}$ assimilation. The \%Ndfa, and $\mathrm{N}$-fixed in response to fertiliser application level is likely caused by a competition for carbon use through sink-source assimilation partitioning. The carbon costs associated with \%Ndfa and $\mathrm{N}$-fixed are substantial and therefore the process occurs and increases at the expense of the biomass accumulation given that $\mathrm{N}_{2}$ fixation is an energetically expensive process [43]. The reduced dependence on symbiotic $\mathrm{N}_{2}$ fixation by intercropped cowpea for its $\mathrm{N}$ nutrition could have been caused by competition for soil $\mathrm{N}$ by the companion crop amaranth. This study corroborates with an earlier study [43] which showed lower \%Ndfa by the intercropped legume due to competition 
from the non-legume crop. In the current study, the control and/or 25\% NPK treatments, though not significantly different from each other, exhibited greater \%Ndfa, whereas the application of $100 \%$ NPK reduced \%Ndfa. With legumes, normally the \%Ndfa is usually enhanced when grown under low soil-N compared to that planted with the supply of high $\mathrm{N}$ [44]. Also, with the application of higher NPK, the legume reduced its symbiotic performance competitive ability. The reduced $\mathrm{N}$ accumulation in the inter-cropping system could have been because the non-legume crop is more competitive for soil $\mathrm{N}$, especially inorganic $\mathrm{N}$ and the competition forces the legume to rely only on $\mathrm{N}_{2}$ fixation, which does not supply as much $\mathrm{N}$ [19]. The addition of higher $\mathrm{N}$ fertilisers can result in a decrease in the richness, diversity, and composition of soil bacterial communities [45]. That being the case, the decreased microbial community, some of which are responsible for solubilisation of soil $\mathrm{N}$, could result in their inefficacy and therefore reduced $\mathrm{N}$ uptake. Understanding cowpea $\mathrm{N}$-accumulation dynamics under varying levels of fertilisation is, therefore, crucial in optimising crop production particularly for the resource-constrained farmers. The cowpea crop tends to depend on atmospheric $\mathrm{N}_{2}$ under limited $\mathrm{N}$ conditions and thus induces $\mathrm{N}$-accumulation in the soil. The findings of this study corroborate with others, such as Singh and Usha [46].

Our results showed that micronutrient ( $\mathrm{Fe}$ and $\mathrm{Zn}$ ) concentrations increased with additional fertiliser application in both cowpea and amaranth across seasons (Table 4). On a crop-by-crop basis, cowpea and amaranth bio-accumulate trace mineral nutrients more in sole cropping when compared to inter-cropping system. Fertiliser application, as well as cropping system, play a key role in micronutrient accumulation of legumes and non-legumes [47]. Cowpea had more micronutrients (Fe and $\mathrm{Zn}$ ) at 25\% NPK fertiliser application level compared to amaranth that increased at the recommended $100 \%$ NPK rate. The behaviour of cowpea agrees with the assumption that legumes have the ability of more nutrient bioaccumulation when compared to non-legumes [48], such as observed in this study. Higher mineral element concentrations in sole cropping is in line with the theory that non-legumes and legumes show capabilities of acquiring Fe and $\mathrm{Zn}$ in an environment with minimal competition from companion plants such as in sole stands relative to intercrops. Similar to other studies such as Dakora and Belane [49], our study found that there is more Fe concentration than $\mathrm{Zn}$ in crops, for example, cowpea and amaranth. The reasons for more iron than zinc on earth generally, is due to phytates bioavailability [50].

Micronutrient considerations become valuable to the vulnerable in society, which include women and children under the age of five [51,52], if they are able to quantify amounts of vegetables to be planted in available land to meet sufficient nutrition, using a concept known as nutritional yield (NY). Nutritional yield is affected, amongst other factors, by fertiliser application as well as inter-cropping. In this study, the NY-values for both seasons and for both crops increased in response to fertiliser application (Table 5). Generally, cowpea had more Fe-NY and Zn-NY, relative to amaranth (Table 5). The NY findings of the combination of the crops in an intercrop showed more Fe-NY and Zn-NY. This shows the advantage of inter-cropping to NY. This study provided insights into the benefits related to NY that can be derived from inter-cropping. The farming community particularly the resource-constrained group could be encouraged to consider more than one crop in limited land area to optimise nutrition in plant tissues, primarily trace elements associated with hidden hunger. The value of the determination of NY with inter-cropping of indigenous vegetables (cowpea and amaranth) on various NPK fertility rates in the soil is to provide more benefits to optimise on limited productive land and eventually minimise trace element deficiency in plant tissues. The variable NY's from cowpea and amaranth especially when combined in an inter-cropping are key in expanding options for the vulnerable in society.

\section{Conclusions}

Cowpea, through symbiotic nitrogen fixation and $\mathrm{N}$-accumulation, was able to contribute $\mathrm{N}$ to the soil. However, the application of fertiliser at $100 \%$ NPK reduced the amounts. Symbiotic nitrogen fixation in cowpea was reduced at $100 \%$ NPK fertiliser levels. However, it was higher at 
lower fertiliser levels such the control ( $0 \%$ NPK) and 25\% NPK. Land utilisation efficiency showed benefits in an intercrop, which resulted in increased accumulation of $\mathrm{Fe}$ and $\mathrm{Zn}$ in plant tissues of both crops, hence increasing nutritional yield. Inter-cropping of cowpea with amaranth contributed to improved NY compared to sole cropping. The study alludes to the importance of minimum resource utilisation to provide more zinc and iron nutrition for the vulnerable in rural communities. The study has implications for improvement of soil fertility in nutrient-poor soils. Through intercropping of cowpea and amaranth, malnutrition in rural resource-poor households can be reduced. Practically, it is recommended, therefore, that smallholder farmers intercrop while reducing fertiliser application by up to $50 \%$ of the recommended dosages. Future recommendations informed by the study is crop rotation to test the residual benefit of cowpea to other vegetables. In addition, nitrogen benefit studies will be important in the future to equate the amount of $\mathrm{N}$-fixed by cowpea to the actual amounts of nitrogen fertiliser to be saved.

Author Contributions: B.M., T.M., A.T.M. and M.F., conceptualised the research project while B.M., executed the experiments and data collection. B.M. and B.N. analysed the data and wrote the original draft manuscript. B.N., M.F., M.N., S.A.K., S.A., T.M., and A.T.M. reviewed and edited the manuscript. T.M., A.T.M. and B.N. did critical review, redrafting and supervised the student's research project. All authors have read and agreed to the published version of the manuscript.

Funding: The study received funding from the Agricultural Research Council under Pipeline Technology Research, which was supported by the Department of Rural Development and Land Reform.

Acknowledgments: We are grateful to FD Dakora for assistance with symbiotic nitrogen fixation and the Agricultural Research Council - Soil, Climate and Water for the analyses of the soil and plant samples.

Conflicts of Interest: The authors declare no conflict of interest.

\section{References}

1. Jones, A.; Breuning-Madsen, H.; Brossard, M.; Dampha, A.; Breuning-Madsen, H.; Brossard, M.; Dampha, A.; Deckers, J.; Dewitte, O.; Le Roux, P.; et al. Soil Atlas of Africa; Publications Office of the European Union: Luxembourg, 2013.

2. Laurie, S.M.; Faber, M.; Claasen, N. Incorporating orange-fleshed sweet potato into the food system as a strategy for improved nutrition: The context of South Africa. Food Res. Int. 2017, 104, 77-85. [CrossRef] [PubMed]

3. Gödecke, T.; Stein, A.J.; Qaim, M.; Gödecke, T.; Stein, A.J.; Qaim, M. The global burden of chronic and hidden hunger: Trends and determinants. Global Food Secur. 2018, 17, 21-29. [CrossRef]

4. Vanlauwe, B.; Descheemaeker, K.; Giller, K.E.; Huising, J.; Merckx, R.; Nziguheba, G.; Zingore, S. Integrated soil fertility management in sub-Saharan Africa: Unravelling local adaptation. Soil 2015, 1, 491-508. [CrossRef]

5. Stewart, Z.P.; Pierzynski, G.M.; Middendorf, B.J.; Prasad, P.V. Approaches to improve soil fertility in sub-Saharan Africa. J. Exp. Bot. 2019, 71, 1-10. [CrossRef]

6. Marschner, P.; Marschner, P. Marschner's Mineral Nutrition of Higher Plants, 3rd ed.; Elsevier Publishers: Amsterdam, The Netherlands, 2012; p. 89.

7. Rietra, R.P.; Heinen, M.; Dimkpa, C.O.; Bindraban, P.S. Effects of nutrient antagonism and synergism on yield and fertiliser use efficiency. Commun. Soil Sci. Plant Anal. 2017, 48, 1895-1920. [CrossRef]

8. Prasad, R.; Shivay, Y.S.; Kumar, D.; Prasad, R.; Shivay, Y.S.; Kumar, D. Agronomic biofortification of cereal grains with iron and zinc. Adv. Agron. 2014, 125, 55-91.

9. Asongu, S.A.; Odhiambo, N.M. Economic development thresholds for a green economy in sub-Saharan Africa. Energy Explor. Exploit. 2019, 0144598719835591. [CrossRef]

10. Bamji, M.S. Food-based approach to combat micronutrient deficiencies. Proc. Indian Natl. Sci. Acad. B Biol. Sci. 2016, 82, 1529-1540.

11. Bioversity International. Diversifying Food and Diets: Using Agricultural Biodiversity to Improve Nutrition and Health, 1st ed.; Routledge: Oxfordshire, UK, 2013. 
12. Zimmerer, K.S.; Judith, A.C.; Steven, J.V. Sustainable smallholder intensification in global change? Pivotal spatial interactions gendered livelihoods and agrobiodiversity. Curr. Opin. Environ. Sustain. 2015, 14, 49-60. [CrossRef]

13. Yahaya, D. Evaluation of Cowpea (Vigna unguiculata (L.) Walp) Genotypes for Drought Tolerance. Ph.D. Thesis, Tennessee State University, Nashville, TN, USA, 2019; pp. 1-24.

14. Owade, J.O.; Abong', G.; Okoth, M.; Mwang'ombe, A.W. A review of the contribution of cowpea leaves to food and nutrition security in East Africa. Food Sci. Nutr. 2019, 6, 1-12. [CrossRef]

15. Jayne, T.S.; Muyanga, M. Land constraints in Kenya's densely populated rural areas: Implications for food policy and institutional reform. Food Secur. 2012, 4, 399-421. [CrossRef]

16. Masvaya, E.N.; Nyamangara, J.; Descheemaeker, K.; Giller, K.E. Is maize-cowpea inter-cropping a viable option for smallholder farms in the risky environments of semi-arid southern Africa? Field Crop. Res. 2017, 209, 73-87. [CrossRef]

17. Ahamefule, E.H.; Peter, P.C. Cowpea (Vigna unguiculata L. Walp) response to phosphorus fertiliser under two tillage and mulch treatments. Soil Tillage Res. 2014, 136, 70-75. [CrossRef]

18. Krause, H.; Faße, A.; Grote, U. Nutrient-Dense Crops for Rural and Peri-Urban Smallholders in Kenya-A Regional Social Accounting Approach. Sustainability 2019, 11, 3017. [CrossRef]

19. Bedoussac, L.; Journet, E.P.; Hauggaard-Nielsen, H.; Naudin, C.; Corre-Hellou, G.; Jensen, E.S.; Justes, E. Ecological principles underlying the increase of productivity achieved by cereal-grain legume intercrops in organic farming. A review. Agron. Sustain. Dev. 2015, 35, 911-935. [CrossRef]

20. Tan, Y.; Hu, F.; Chai, Q.; Li, G.; Coulter, J.A.; Zhao, C.; Yin, W. Expanding row ratio with lowered nitrogen fertilisation improves system productivity of maize/pea strip intercropping. Eur. J. Agron. 2020, 113, 125986. [CrossRef]

21. Zuo, Y.; Zhang, F. Iron and zinc biofortification strategies in dicot plants by intercropping with gramineous species. A review. Agron. Sustain. Dev. 2009, 29, 63-71. [CrossRef]

22. Bumgarner, N.R.; Scheerens, J.C.; Kleinhenz, M.D. Nutritional yield: A proposed index for fresh food improvement illustrated with leafy vegetable data. Plant Food Hum. Nutr. 2012, 67, 215-222. [CrossRef]

23. Mndzebele, B.; Ncube, B.; Fessehazion, M.; Mabhaudhi, T.; Amoo, S.; du Plooy, C.; Modi, A. Effects of Cowpea-Amaranth Intercropping and Fertiliser Application on Soil Phosphatase Activities, Available Soil Phosphorus, and Crop Growth Response. Agronomy 2020, 10, 79. [CrossRef]

24. Beletse, Y.; du Plooy, I.; Jansen van Rensburg, W. Water Requirement of Selected African Leafy Vegetables. In Nutritional Value and Water Use of African Leafy Vegetables for Improved Livelihoods; WRC TT535/12; Oelofse, A., Van Averbeke, W., Eds.; Water Research Commission: Pretoria, South Africa, 2012.

25. Herridge, D.F.; Peoples, M.B. Timing of xylem sampling for ureide analysis of nitrogen fixation. Plant Soil 2002, 238, 57-67. [CrossRef]

26. Shearer, G.; Kohl, D.H. $\mathrm{N}_{2}$-fixation in field settings: Estimations based on natural ${ }^{15} \mathrm{~N}$ abundance. Funct. Plant Biol. 1986, 13, 699-756.

27. Zasoski, R.J.; Burau, R.G. A rapid nitric acid digestion method for multi-element tissue analysis. Commun. Soil Sci. Plant Anal. 1977, 8, 425-436. [CrossRef]

28. Pausch, R.C.; Mulchi, C.L.; Lee, E.H.; Meisinger, J.J. Use of ${ }^{13} \mathrm{C}$ and ${ }^{15} \mathrm{~N}$ isotopes to investigate $\mathrm{O}_{3}$ effects on $\mathrm{C}$ and $\mathrm{N}$ metabolism in soybeans. Part II. Nitrogen uptake, fixation, and partitioning. Agric. Ecosyst. Environ. 1996, 60, 61-69. [CrossRef]

29. Young, E.G.; Conway, C.F. On the estimation of allantoin by the Rimini-Schryver reaction. J. Biol. Chem. 1942, 142, 839-853.

30. Unkovich, M.; Herridge, D.A.V.I.D.; Peoples, M.; Cadisch, G.; Boddey, B.; Giller, K.; Chalk, P. Measuring Plant-Associated Nitrogen Fixation in agricultural Systems; Australian Centre for International Agricultural Research (ACIAR): Canberra, Australia, 2008.

31. Cataldo, D.A.; Maroon, M.; Schrader, L.E.; Youngs, V.L. Rapid colorimetric determination of nitrate in plant tissue by nitration of salicylic acid. Commun. Soil Sci. Plant 1975, 6, 71-80. [CrossRef]

32. Leidi, E.O.; Rodríguez-Navarro, D.N. Nitrogen and phosphorus availability limit $\mathrm{N}_{2}$ fixation in bean. New Phytol. 2000, 147, 337-346. [CrossRef]

33. Zhihui, W.E.N.; Jianbo, S.H.E.N.; Blackwell, M.; Haigang, L.I.; Bingqiang, Z.H.A.O.; Huimin, Y.U.A.N. Combined applications of nitrogen and phosphorus fertilisers with manure increase maize yield and nutrient uptake via stimulating root growth in a long-term experiment. Pedosphere 2016, 26, 62-73. 
34. Mndzebele, B.M.; Dakora, F.D. Plant growth and $\mathrm{N}_{2}$ fixation in Cyclopia longifolia (Vogel L.) supplied with mineral nutrients in pot and field experiments. S. Afr. J. Bot. 2017, 110, 97-102. [CrossRef]

35. Ike, K.A.; Nwaigbo, L.C.; Obasi, C.P.; Olanite, J.A.; Chilaka, O.M. Effect of Fertilizer LEVEL and Intercropping with Bambara Nut (Vigna subterranea) on the Growth and Herbage Yield of Maize. Pastures in Integrated Crop/Livestock Production Systems; University of Kentucky: Lexington, KY, USA, 2020; pp. 974-975.

36. Mitran, T.; Meena, R.S.; Lal, R.; Layek, J.; Kumar, S.; Datta, R. Role of Soil Phosphorus on Legume Production. In Legumes for Soil Health and Sustainable Management; Springer: Berlin/Heidelberg, Germany, 2018; pp. 487-510.

37. Clayton, G.W.; Rice, W.A.; Lupwayi, N.Z.; Johnston, A.M.; Lafond, G.P.; Grant, C.A.; Walley, F. Inoculant formulation and fertiliser nitrogen effects on field pea: Crop yield and seed quality. Can. J. Plant Sci. 2004, 84, 89-96. [CrossRef]

38. Ghosh, P.K.; Bandyopadhyay, K.K.; Manna, M.C.; Mandal, K.G.; Misra, A.K.; Hati, K.M. Comparative effectiveness of cattle manure. Poultry manure. Phosphocompost and fertiliser-NPK on three cropping systems in vertisols of semi-arid tropics. II. Dry matter yield. Nodulation, chlorophyll content and enzyme activity. Bioresour. Technol. 2004, 95, 85-93. [CrossRef]

39. Nyoki, D.; Ndakidemi, P.A. Rhizobium inoculation reduces $\mathrm{P}$ and $\mathrm{K}$ fertilisation requirement in corn-soybean inter-cropping. Rhizosphere 2018, 5, 51-56. [CrossRef]

40. Ofori, F.; Stern, W. Cereal-legume intercropping systems. Adv. Agron. 1987, 41, 41-90.

41. Oroka, F.O.; Omoregie, A.U. Competition in a rice-cowpea intercrop as affected by nitrogen fertiliser and plant population. Sci. Agric. 2007, 64, 621-629. [CrossRef]

42. Anglade, J.; Billen, G.; Garnier, J. Relationships for estimating $\mathrm{N}_{2}$ fixation in legumes: Incidence for $\mathrm{N}$ balance of legume-based cropping systems in Europe. Ecosphere 2015, 6, 1-24. [CrossRef]

43. Fan, F.; Zhang, F.; Song, Y.; Sun, J.; Bao, X.; Guo, T.; Li, L. Nitrogen fixation of faba bean (Vicia faba L.) interacting with a non-legume in two contrasting inter-cropping systems. Plant Soil 2006, 283, 275-286. [CrossRef]

44. Corre-Hellou, G.; Brisson, N.; Launay, M.; Fustec, J.; Crozat, Y. Effect of root depth penetration on soil nitrogen competitive interactions and dry matter production in pea-barley intercrops given different soil nitrogen supplies. Field Crop. Res. 2007, 103, 76-85. [CrossRef]

45. Zhou, H.; Zhang, D.; Wang, P.; Liu, X.; Cheng, K.; Li, L.; van Zwieten, L. Changes in microbial biomass and the metabolic quotient with biochar addition to agricultural soils: A Meta-analysis. Agric. Ecosyst. Environ. 2017, 239, 80-89. [CrossRef]

46. Singh, B.; Usha, K. Nodulation and symbiotic nitrogen fixation of cowpea genotypes as affected by fertiliser nitrogen. J. Plant Nutr. 2003, 26, 463-473. [CrossRef]

47. Nyoki, D.; Ndakidemi, P.A. Intercropping system, rhizobia inoculation, phosphorus and potassium fertilisation: A strategy of soil replenishment for improved crop yield. Int. J. Curr. Microbiol. Appl. Sci. 2016, 5, 504-522. [CrossRef]

48. Wortmann, C.S.; Dicko, M.K.; Maman, N.; Senkoro, C.J.; Tarfa, B.D. Fertiliser Application Effects on Grain and Storage Root Nutrient Concentration. Agron. J. 2018, 110, 2619-2625. [CrossRef]

49. Dakora, F.D.; Belane, A.K. Evaluation of Protein and Micronutrient Levels in Edible Cowpea (Vigna unguiculata L. Walp.) Leaves and Seeds. Front. Sustain. Food Syst. 2019, 3, 70. [CrossRef]

50. Dary, O.; Hurrell, R. Guidelines on food Fortification with Micronutrients; World Health Organization, Food and Agricultural Organization of the United Nations: Geneva, Switzerland, 2006.

51. Arimond, M.; Wiesmann, D.; Becquey, E.; Carriquiry, A.; Daniels, M.; Deitchler, M. Dietary Diversity as a Measure of the Micronutrient Adequacy of Women's Diets in Resource-Poor Areas: Summary of Results from Five Sites; FANTA II Project: Washington, DC, USA, 2011.

52. Black, R.E.; Victora, C.G.; Walker, S.P.; Bhutta, Z.A.; Christian, P.; de Onis, M. Maternal and child undernutrition and overweight in low-income and middle-income countries. Lancet 2013, 382, 427-451. [CrossRef]

(C) 2020 by the authors. Licensee MDPI, Basel, Switzerland. This article is an open access article distributed under the terms and conditions of the Creative Commons Attribution (CC BY) license (http://creativecommons.org/licenses/by/4.0/). 